\title{
Metasíntesis sobre la narrativa educativa durante la pandemia por COVID-19
}

\section{A meta-synthesis regarding the educational narrative during the COVID-19 Pandemic}

DOI: https://doi.org/ 10.32870/dse.v0i22.849

\section{Alexandro Escudero-Nahón*}

\begin{abstract}
Resumen
La pandemia por COVID-19 obligó al Sistema Educativo Nacional mexicano a suspender abruptamente las clases presenciales y realizar actividades educativas alternativas para continuar con la docencia. La mayoría de las instituciones respondieron reactivamente porque muy pocas cuentan con planes de continuidad académica. Esto provocó que algunos organismos mexicanos de reconocido prestigio crearan foros virtuales y seminarios para analizar los problemas educativos que planteó esta contingencia sanitaria y los desafíos que se enfrentarían en el periodo postCOVID. Se realizó una metasíntesis sobre la narrativa educativa que desarrollaron las y los especialistas de cinco organismos mexicanos especializados en educación y en tecnología educativa. Se revisaron 81 páginas web oficiales de Instituciones de Educación Superior y 31 eventos virtuales (foros y seminarios) con aproximadamente 3,564 minutos de grabación. Los resultados sugieren que existe un amplio consenso respecto a analizar los efectos educativos de esta contingencia desde un criterio cronológico: antes, inicio, durante y después de la pandemia. Se concluyó que la contingencia de salud no provocó nuevos problemas educativos, sino que subrayó problemas de desigualdad y deficiencias educativas preexistentes. Sin embargo, se detectó la necesidad de diseñar planes de continuidad académica y políticas públicas con la participación de las familias.
\end{abstract}

Palabras clave: educación superior - contingencia sanitaria - investigación documental - metasíntesis continuidad académica.

\begin{abstract}
The COVID 19 pandemic forced the Mexican National Education System to abruptly suspend face-to-face classes and use alternative educational activities to continue teaching. Most institutions responded reactively because very few have plans for academic continuity. This caused some prestigious Mexican organizations to create virtual forums and seminars to analyze the educational problems posed by this health contingency and the challenges they would face in the post-COVID 19 period. A meta-synthesis was carried
\end{abstract}

* Doctor en Educación. SNI Nivel 1. Cuerpo Académico Innovación Educativa y Tecnología. Dirige el Proyecto Transdigital, Iniciativa Ciudadana para la Difusión de la Ciencia. Coordinador del Comité de Investigación y Posgrados en Tecnología Educativa de la RedLaTE México, Red Temática CONACyTEs. Profesor investigador de tiempo completo y coordinador del Doctorado en Tecnología Educativa en la Facultad de Informática de la Universidad Autónoma de Querétaro. México. alexandro.escudero@uaq.mx 
out regarding the educational narrative developed by the specialists of five Mexican organizations specialized in education and educational technology. 81 official websites of Higher Education Institutions and 31 virtual events (forums and seminars), including approximately 3,564 minutes of recording, were reviewed. The findings suggest that there is broad consensus on analyzing the educative effects of this contingency from a chronological point of view: before, beginning, during and after the COVID-19 pandemic. It was concluded that the health contingency did not cause new educational problems, but rather underlined previously existing inequality problems and educational deficiencies. However, we detected the need to have parents participate in the design of academic continuity plans as well as public policies with a broad participation of families.

Keywords: higher education - health contingency - documentary research - meta-synthesis - academic continuity.

\section{Introducción}

La actual pandemia por COVID-19 afectó la educación de millones de personas en el mundo porque las instituciones educativas tuvieron que cerrar sus instalaciones como medida preventiva ante la propagación del virus SARS-CoV-2. Se estima que aproximadamente 1,268'164,088 estudiantes dejaron de asistir a clases presenciales en todo el planeta (UNESCO, 2020b). Lo anterior representa $72.4 \%$ del total de alumnos matriculados mundialmente. En la educación superior, fueron afectadas más de 25 mil universidades con más de 200 millones de estudiantes (UNESCO, 2020a). En México, la pandemia por COVID-19 afectó a casi 40.7 millones de personas (37.7 millones de estudiantes; 2 millones de docentes; y 999,835 personas que realizan actividades no docentes), que conforman al Sistema Educativo Nacional (SEN). En la educación superior mexicana fueron afectadas 6,404 Instituciones de Educación Superior (IES) (de las cuales 2,455 son públicas; 3,949 son privadas) que albergan a casi 5.3 millones de personas (4.7 millones estudiantes; 429,495 docentes; y 188,646 personas que realizan actividades no docentes). Lo anterior equivale a $4.2 \%$ de la población mexicana (SEP, 2020).

Ante este problema de alcance global, la Organización de las Naciones Unidas para la Educación, la Ciencia y la Cultura (UNESCO, por las siglas en inglés) registró puntualmente en su sitio web oficial algunas prácticas educativas desarrolladas internacionalmente (UNESCO, 2020b). En México, algunos organismos de reconocido prestigio, como la Asociación Nacional de Universidades e Instituciones de Educación Superior (ANUIES), el Comité de Tecnologías de Información y Comunicación de la ANUIES (ANUIES-TIC), la Red Temática Mexicana para el Desarrollo e Incorporación de Tecnología Educativa (RedLaTE), la Corporación Universitaria para el Desarrollo de Internet (CUDI) y el Consejo Mexicano de Investigación Educativa (COMIE), crearon foros virtuales y seminarios para analizar los problemas educativos que planteó esta contingencia sanitaria y los desafíos que serán enfrentados en el periodo postCOVID.

Diálo@os sobre Educación año 12 | número 22 | enero-junio 2021 | ISSN 2007-2171 
La ANUIES es una asociación no gubernamental fundada en 1950. Destaca porque ha participado en la formulación de programas, planes y políticas nacionales sobre la educación superior mexicana. Agremia a las principales instituciones de educación superior de México y produce información relevante sobre los ámbitos de la docencia, la investigación y la extensión de la cultura y los servicios. Actualmente, está conformada por 197 universidades e instituciones de educación superior, tanto públicas como particulares de todo el país (ANUIES, 2020c).

Además, el Comité de Tecnologías de Información y Comunicación de la ANUIES (ANUIESTIC) tiene el objetivo de asesorar y proponer a las IES asociadas sobre temas que se consideren oportunos en el ámbito de las tecnologías de la información y las comunicaciones para mejorar la calidad, la eficacia y la eficiencia de los servicios académico-administrativos proporcionados por las IES, así como fomentar, promover y liderar la cooperación entre sus miembros (ANUIES, 2020b).

La RedLaTE es una red temática reconocida por el Consejo Nacional de Ciencia y Tecnología (CONACyT) desde el año 2015 y tiene el compromiso de asesorar, a quien así lo solicite, en materia de diseño de políticas educativas, culturales y científico-tecnológicas. Esta red está orientada a la investigación, innovación y desarrollo tecnológico especializado, así como a la formulación de iniciativas legislativas asociadas a temas de informática educativa, cómputo educativo, robótica pedagógica y, en general, a todas las áreas afines a la tecnología educativa (RedLaTE, 2020).

La CUDI es una asociación civil sin fines de lucro que gestiona la Red Nacional de Educación e Investigación (RNEI) para promover el desarrollo tecnológico de México y aumentar la sinergia entre sus integrantes. Fue fundada en abril de 1999 y tiene como principal objetivo fomentar la colaboración en proyectos de investigación y educación entre sus miembros (CUDI, 2020).

Finalmente, el COMIE es una asociación civil constituida el 23 de septiembre de 1993, que reúne investigadores del más alto nivel con el objetivo principal de promover la investigación educativa dentro de estándares científicos de calidad. A partir de este objetivo, ha contribuido de manera consistente y continua a la identificación de los principales problemas educativos de México, así como a la generación de los conocimientos necesarios para su solución (COMIE, 2020a).

La información técnica y documental proporcionada por todos estos organismos fue pertinente, actual y útil para los fines de este estudio.

El objetivo de esta investigación documental fue analizar la narrativa que desarrollaron las y los especialistas de estos organismos sobre los problemas educativos que planteó esta contingencia sanitaria y los desafíos que se enfrentarían en el periodo postCOVID. La información obtenida alude, de forma general, a todos los niveles educativos en México.

\section{Método}

Este estudio documental fue conducido con el método llamado metasíntesis. La metasíntesis es un estudio propiamente cualitativo. Por eso, se abordó la información de manera inductiva, 
es decir, sin categorías de análisis predefinidas, y se trataron los datos desde un punto de vista hermenéutico para construir categorías de análisis adecuadas al problema en cuestión.

La elección del método fue pertinente debido a dos situaciones. En primer lugar, puesto que la pandemia por COVID-19 es muy reciente, aún no se ha publicado suficiente literatura científica sobre investigación empírica al respecto; esta situación dificultó la aplicación de otro tipo de estudios de carácter cuantitativo, como el meta-análisis (Leary, Walker, 2018). En segundo lugar, debido a que la estrategia oficial del Gobierno de la República mexicana para evitar los contagios del virus SARS-CoV-2, denominada "sana distancia", comprendió varias medidas para fomentar el confinamiento en los hogares (Gobierno de México, 2020), no fue posible realizar un estudio de campo. La única opción para obtener información pertinente fue visitar sitios y páginas web oficiales de organismos especializados. La pandemia por COVID 19 provocó una polémica educativa, pero al mismo tiempo impidió la reunión física de las y los especialistas. Por eso, diversos organismos especializados en la investigación educativa realizaron eventos virtuales para presentar información sintética y útil. Se realizaron páginas web, foros y seminarios virtuales donde las ponencias presentadas eran una síntesis de información relevante. Esta metasíntesis, reunió, analizó y después sintetizó la narrativa educativa que desarrollaron las y los especialistas en esos eventos virtuales. El resultado fue la comprensión integral de diversas narrativas sobre un problema educativo complejo (Finfgeld-Connett, 2016, 2018).

El procedimiento de investigación se realizó en cuatro fases (figura 1).

\section{Pregunta de investigación}

En primer lugar, se planteó la pregunta de investigación: ¿qué desafíos educativos surgieron debido a la contingencia sanitaria por la pandemia COVID-19 y qué problemas educativos surgirían en el periodo postCOVID?

Figura 1. Procedimiento de investigación en cuatro fases

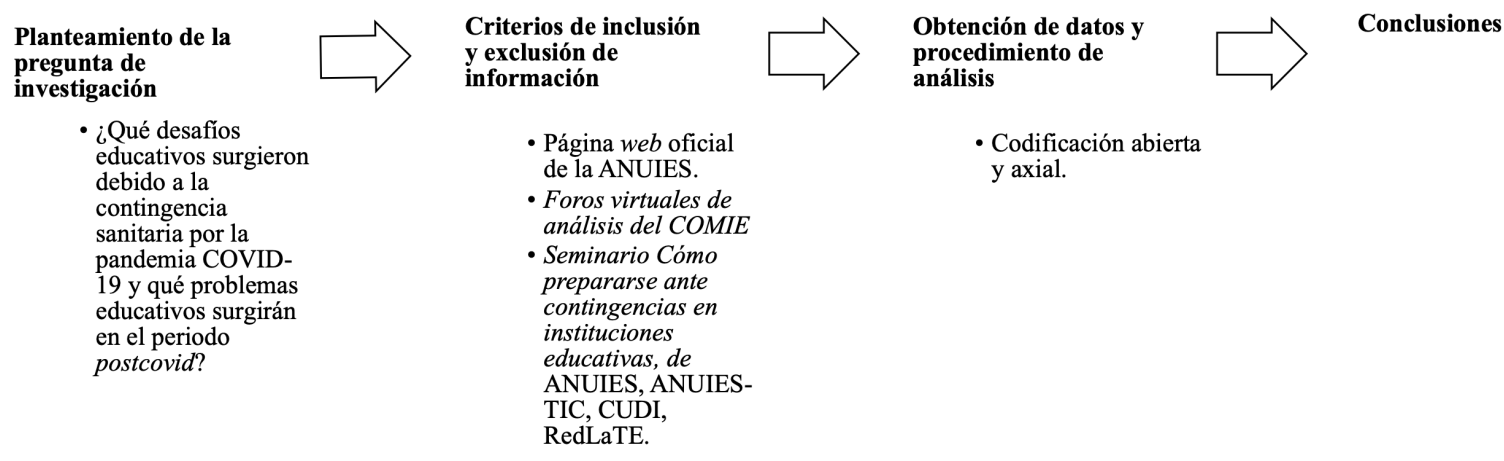

Fuente: elaboración propia.

DiáloQos 


\section{Criterios de inclusión y exclusión de la información}

El criterio más importante para incluir o excluir información en la metasíntesis estuvo definido por la pregunta de investigación y la pertinencia, actualidad y utilidad de dicha información (Finlayson, Dixon, 2008). Por su carácter inductivo, esta metasíntesis no contempló una muestra representativa de documentos, sino la pertinencia de una muestra teórica (Breckenridge, Jones, 2009; Butler et al., 2018). Se obtuvo información de las y los especialistas de cinco organismos mexicanos especializados en educación y en tecnología educativa. Se revisaron 81 páginas web oficiales de IES y 31 eventos virtuales (foros y seminarios) con aproximadamente 3,564 minutos de grabación (tabla 1).

\begin{tabular}{|c|c|c|}
\hline \multicolumn{3}{|c|}{ Tabla 1. Fuentes de información y documentos analizados } \\
\hline Fuente de información & Organización & Documentación analizada \\
\hline $\begin{array}{l}\text { Acciones ante la contin- } \\
\text { gencia y la continuidad de } \\
\text { las universidades educa- } \\
\text { tivas }\end{array}$ & ANUIES & $\begin{array}{l}81 \text { links sobre actividades educativas o pla- } \\
\text { nes de continuidad académica de } 51 \text { IES; se } \\
\text { desestimaron } 37 \text { links por no ser pertinen- } \\
\text { tes al análisis (anexo } 1 \text { ). }\end{array}$ \\
\hline Foro virtual de análisis & COMIE & $\begin{array}{l}16 \text { videos correspondientes a las sesiones } \\
\text { virtuales de análisis, con una duración total } \\
\text { de } 2,032 \text { minutos (anexo } 2 \text { ). }\end{array}$ \\
\hline $\begin{array}{l}\text { Seminario: Cómo prepa- } \\
\text { rarse ante contingencias } \\
\text { en instituciones educati- } \\
\text { vas }\end{array}$ & $\begin{array}{c}\text { ANUIES, ANUIES-TIC, } \\
\text { CUDI y RedLaTE }\end{array}$ & $\begin{array}{l}15 \text { videos correspondientes a los semina- } \\
\text { rios, con una duración total de 1,532 minu- } \\
\text { tos (anexo 3). }\end{array}$ \\
\hline
\end{tabular}

\section{Obtención de información y análisis de los datos}

El procedimiento para obtener la información fue sensible al contexto (Finfgeld-Connett, 2010). En primer lugar, estuvo sujeto a la evolución de la pandemia y a las decisiones que tomaron las autoridades educativas mexicanas. A partir del 20 de marzo de 2020 se suspendieron las clases presenciales en la educación básica y media superior por indicaciones del director de la Secretaría de Educación Pública (SEP). La mayoría de las IES también suspendió las clases presenciales a partir de esa fecha, e incluso, algunos días antes. Durante abril, mayo y junio de 2020 se realizaron varios foros virtuales y seminarios sobre los desafíos que impuso la interrupción de las clases presenciales y el desarrollo de actividades docentes alternativas. Por eso, el periodo durante el cual se realizó la búsqueda de información fue del 20 de junio al 20 de julio de 2020.

Respecto a las Acciones ante la contingencia y la continuidad de las universidades educativas, se revisaron 81 links que dirigen las acciones educativas realizadas durante la contingencia sanitaria por 51 IES mexicanas (ANUIES, 2020a). Se detectó que no todas esas acciones son de carácter educativo; algunas tienen el objetivo de informar sobre la pandemia por COVID-19. 
Muy pocas de esas acciones son, propiamente, planes de continuidad académica, la mayoría son repositorios de recursos digitales y comunicados oficiales para remediar la falta de ese tipo de planes. Además, muchos links conducen a páginas restringidas con un nombre de usuario y contraseña. Algunos links, en realidad, duplicaban información, puesto que se presentaron sitios y micrositios por separado, cuando un solo link contenía la información del anterior. Varias de las acciones registradas por las IES se dirigen a las plataformas de educación virtual o a distancia que operan habitualmente; es decir, no son páginas web diseñadas específicamente para la continuidad académica tras la contingencia sanitaria. La metasíntesis solo consideró las acciones de carácter educativo que presentaron información de carácter público y con información específica sobre actividades educativas o planes de continuidad académica. En total se revisaron 44 links (anexo 1).

Respecto al Foro virtual de análisis, se revisaron 16 videos correspondientes a las sesiones virtuales de análisis, con una duración total de 2,032 minutos (COMIE, 2020b). Los foros analizaron los problemas provocados por la pandemia por COVID-19 en todos los niveles educativos y en diversos subtemas educativos (anexo 2).

Respecto al Seminario Cómo prepararse ante contingencias en instituciones educativas, se revisaron 15 videos, con una duración total de 3,564 minutos (ANUIES et al., 2020). En este seminario se presentaron varias aplicaciones digitales educativas y los planes educativos de 21 IES: Universidad Autónoma de Baja California, Universidad de Guadalajara, Universidad Autónoma Metropolitana, Instituto Tecnológico y de Estudios Superiores de Monterrey, Instituto Politécnico Nacional, Instituto Tecnológico Autónomo de México, Universidad La Salle Ciudad de México, Universidad Nacional Autónoma de México, Tecnológico Nacional de México, Universidad Pedagógica Nacional, Universidad Autónoma de Yucatán, Universidad Autónoma del Carmen, Universidad Veracruzana, Universidad Autónoma de Nuevo León, Universidad de las Américas Puebla, Universidad Autónoma del Estado de México, Instituto Tecnológico de Sonora, Universidad Autónoma de Tamaulipas, Universidad Anáhuac, El Colegio de México y Universidad de Colima (anexo 3).

El procesamiento de la información se realizó con un método de codificación basado en la Teoría Fundamentada: codificación abierta y codificación axial. Este método de codificación es habitual en las investigaciones con enfoque cualitativo; tiene el objetivo principal de describir detalladamente el principal problema de un grupo de personas y qué hicieron para resolverlo (Flick, 2015; Guetterman et al., 2017). El objetivo de la codificación fue generar una descripción detallada sobre la narrativa que las y los especialistas produjeron debido a un problema emergente: los desafíos educativos que surgieron debido a la contingencia sanitaria por la pandemia COVID-19 y los problemas educativos que surgirán en el periodo postCOVID. La codificación abierta hizo referencia a abrir la realidad; es decir, obtuvo información de varias fuentes y le dio sentido a través de categorías conceptuales. Una vez que se obtuvo una lista de categorías con-

Diólo@os sobre Educación TEMAS ACTUALES EN INVESTIGACTON EDCA año 12 | número 22 | enero-junio 2021 | ISSN 2007-2171 
ceptuales, se procedió a construir la codificación axial, que fue una representación gráfica sobre cómo se relacionan esas categorías conceptuales entre sí.

\section{Conclusiones}

Las conclusiones se presentan, propiamente, en la cuarta sección de este texto.

\section{Resultados}

Al codificar de manera abierta la narrativa de las y los especialistas, se construyó una lista de 89 categorías conceptuales. Al construir la codificación axial, estas categorías se redujeron, relacionaron y ordenaron con base en un criterio de análisis que gozó de amplio consenso entre las y los especialistas: un criterio cronológico (antes, inicio, durante y después de la pandemia por COVID-19). La narrativa de las y los especialistas hizo alusión, recurrentemente, a dos ámbitos simbólicos problemáticos: 1) Las instituciones educativas, encarnadas en las instalaciones físicas de las escuelas, bachilleratos o universidades; 2) Las familias, encarnadas en los espacios físicos de las casas. Por eso, las categorías conceptuales señalaron constantemente la tensión que prevaleció entre ambos espacios físicos y simbólicos al inicio y durante la contingencia sanitaria; no obstante, también apuntaron que antes de la contingencia esas mismas tensiones existían pero estaban soterradas. Para efectos de la descripción detallada de la metasíntesis se dividió la codificación axial en cuadrantes, donde se detallan los principales problemas educativos y familiares experimentados antes, al inicio, durante y después de la contingencia sanitaria (figura 2).

Figura 2. Principales problemas de las instituciones educativas y las familias durante la contingencia sanitaria por COVID-19

\begin{tabular}{lll}
\hline Fase & Familias \\
Anstituciones Deficiencias & B) Desigualdades \\
Inicio & C) Desconciertos & D) Reorganizaciones \\
Durante & E) Adaptaciones & F) Agobios \\
Después & G) Recusamientos & H) (des)Involucramientos
\end{tabular}

Fuente: elaboración propia. 
A) Deficiencias: los principales problemas educativos antes de la contingencia sanitaria por COVID-19. La contingencia sanitaria no provocó ningún problema radicalmente nuevo, pero permitió ver con dolorosa nitidez los problemas educativos que han existido desde hace décadas. El hecho de que esas deficiencias educativas sean de carácter crónico llamó la atención sobre la incapacidad o la negligencia del SEN para resolverlos de manera inmediata tras esta contingencia sanitaria. De acuerdo con la narrativa de las y los especialistas, se anticipó que el regreso a la nueva normalidad será, en realidad, el regreso a una anormalidad educativa crónica.

B) Desigualdades: los principales problemas familiares antes de la contingencia sanitaria por COVID-19. La contingencia destacó que no hay participación de las familias en la educación formal debido a que no existen políticas públicas al respecto. Existe un amplio consenso entre las y los especialistas respecto a que la poca participación parental en la educación formal tiene efectos indeseables, como desencuentros y enfrentamientos entre docentes y familiares. Esta situación se radicalizó durante el periodo de confinamiento porque la colaboración familiar fue fundamental para asistir y legitimar a las y los docentes.

C) Desconciertos: los principales problemas educativos al inicio de la contingencia sanitaria por COVID-19. La narrativa de las y los especialistas coincidió en que al momento de interrumpir las clases presenciales e intentar continuar con las actividades docentes, solo se logró enseñanza remota de emergencia. De ninguna manera se realizó educación a distancia o educación virtual, porque estas dos modalidades educativas requieren, propiamente, planeación, diseño de objetos de aprendizaje digitales adecuados a la tecnología digital, vías de comunicación sofisticadas, procesos de automatriculación, trámites escolares autogestivos y criterios para evaluar la docencia y el aprendizaje. Si acaso, las y los docentes más responsables, creativos y heroicos solo desarrollaron enseñanza remota de emergencia.

Este tipo de enseñanza develó que, ante el desconcierto, tanto el alumnado como las y los docentes experimentaron problemas similares: desconocimiento del uso educativo de aplicaciones digitales, problemas de conectividad, dificultades de comunicación, distracciones domésticas, problemas para negociar los espacios físicos en casa, incapacidad para gestionar el tiempo y para organizarse de acuerdo con los principios del teletrabajo.

El hecho de que las y los docentes no estuvieran preparados para desarrollar sus cursos con un plan de continuidad académica provocó que tuvieran una visión muy limitada de las opciones educativas que ofrece la tecnología digital. De tal manera que la docencia se realizó bajo condiciones y estándares personales de cada docente, y no bajo un modelo educativo. Esta situación provocó varios efectos perniciosos, pero el más grave fue que se acentuaron las brechas de desigualdad del alumnado, porque muchas de las decisiones docentes tuvieron como objetivo restituir cierta seguridad y confianza de quienes impartieron las clases y no necesariamente de quienes las recibieron. La narrativa de las y los especialistas coincide en que esas decisiones docentes fueron desafortunadas pero comprensibles, considerando que las autoridades mos- 
traron una incapacidad institucional para alinear rápidamente a toda la comunidad educativa sobre un eje de acción claro. De hecho, las autoridades reaccionaron emitiendo comunicados y directrices oficiales espontáneas, contradictorias, ambiguas, que constantemente fueron revisando y ajustando.

Estrictamente, en términos de aprendizaje, el problema principal radicó en el hecho de que la burocracia educativa intentó trasponer su dinámica a los hogares. Por eso, aun cuando la contingencia sanitaria fue un escenario inédito e incierto para toda la comunidad educativa, las autoridades mantuvieron el carácter formal: las clases síncronas, el pase de lista, los calendarios escolares fijos, la atención en el material educativo como principal medio para enseñar contenidos formales (que eran irrelevantes ante la situación sanitaria), las actividades memorísticas, etcétera. En cambio, se desestimó la oportunidad de aprender otros temas más significativos con métodos basados en el desarrollo de las habilidades blandas, como el diálogo, la cooperación o el cuidado del otro.

De acuerdo con la narrativa de las y los especialistas, no se debe perder de vista que al inicio de la contingencia educativa solo se obtuvieron datos, muy desarticulados, de $40 \%$ de las y los alumnos matriculados en el SEN. Esta cifra tan baja despertó dos preocupaciones: 1) Quienes no pudieron conectarse seguramente son un grupo en situación de vulnerabilidad, no solo educativa sino sanitariamente; 2) Quienes no quisieron conectarse, demostraron su desafección por los procesos educativos formales.

D) Reorganizaciones: los principales problemas familiares al inicio de la contingencia sanitaria por COVID-19. Las desigualdades sociales previas a la contingencia sanitaria provocaron que no hubiera confinamientos homogéneos. En otras palabras, las experiencias familiares sobre la enseñanza remota de emergencia estuvieron matizadas por el nivel socioeconómico y cultural de cada caso en cuestión. Esta situación fue importante porque, debido a que las instituciones no contaban con planes de continuidad académica, cada docente utilizó la aplicación digital de su preferencia. Esto supuso una sobrecarga de trabajo en el alumnado que repercutió en las familias: el alumnado tuvo que aprender a usar todas las aplicaciones digitales de todos sus docentes en poco tiempo; para lograrlo, requirió dispositivos digitales y, dependiendo del nivel socioeconómico y cultural, este proceso fue más o menos costoso y traumático.

En el caso específico de los estudiantes de educación básica y media, esa carga de trabajo y esos requerimientos de equipo se extendieron a los parientes. En el peor de los casos, los parientes se convirtieron en asistentes de los profesores y también tuvieron que aprender a usar todas las aplicaciones digitales de todos los docentes en poco tiempo. Esta situación provocó tensiones económicas, de relación humana y de comunicación en el interior de las familias, pero tuvo efectos más adversos en las familias cuyos miembros coexisten en un mismo espacio físico, pero no mantienen una relación relativamente armoniosa.

E) Adaptaciones: Ios principales problemas educativos durante la contingencia sanitaria por COVID-19. Los planes de continuidad académica describen las aplicaciones digitales que la ins- 
titución pone a disposición de la comunidad educativa, la normatividad para utilizarlas, las vías de comunicación institucionales y los criterios para evaluar el aprendizaje y la docencia. Pocas IES contaban con estos planes al inicio de la contingencia sanitaria y solo algunas los fueron construyendo durante la misma. Por eso, los departamentos técnicos, informáticos o de educación a distancia de las IES se vieron obligados a abrir cientos o miles de aulas virtuales con el objetivo de emular las clases presenciales en espacios virtuales. Este proceso fue tortuoso y relativamente lento.

De acuerdo con la narrativa de las y los especialistas, lo anterior obligó a que cada docente resolviera su enseñanza remota de emergencia con los recursos personales disponibles. Esto supuso el uso espontáneo de una diversidad abigarrada de aplicaciones y de las redes sociales, pero con pocas habilidades para obtener beneficios educativos. En general, ni el profesorado ni el alumnado mostraron habilidades para usar las redes sociales con fines educativos. Aunque estas fallidas adaptaciones de las aplicaciones digitales y las redes sociales paliaron los problemas que planteó la docencia de asignaturas teóricas, fueron insuficientes para cumplir con los objetivos de ciertas asignaturas eminentemente prácticas, técnicas o tecnológicas. En este caso, no hay evidencias de que estas asignaturas se hayan desarrollaron adecuadamente.

Debido a que no hubo criterios institucionales para evaluar la docencia, las y los profesores debieron demostrar que realizaban su labor docente con evidencias de diversos tipos, como el pase de lista, los horarios de clase fijos, los calendarios inflexibles y un número excesivo de tareas. Estas evidencias sirvieron para cumplir con la dimensión administrativa de la docencia, pero no para demostrar el aprendizaje del alumnado. Debido a que tampoco hubo criterios institucionales para evaluar el aprendizaje en situación de enseñanza remota de emergencia, algunos tipos de evaluación no se cumplieron a cabalidad y otros, definitivamente, no se llevaron a cabo: la evaluación sumativa, que mide el producto del aprendizaje, fue una simulación debido a que no se realizó de manera adecuada a la enseñanza remota; la evaluación formativa, que mide el progreso del aprendizaje, se exageró con un sinnúmero de tareas; no hubo procesos serios de autoevaluación ni de coevaluación.

El hecho de que las y los docentes se vieran envueltos súbitamente en ambientes de teletrabajo, pero sin las condiciones óptimas del mismo, es decir, sin respeto por sus periodos de descanso, sin insumos digitales adecuados, sin criterios de evaluación de su labor, provocó dos situaciones. Por un lado, desarrollaron soluciones creativas al desafío que impuso la contingencia sanitaria (pero no existen registros institucionales al respecto, solo anécdotas). Por otro lado, se formaron una idea errónea sobre las posibilidades que brindan las nuevas modalidades educativas que utilizan tecnología digital. En general, tanto el alumnado como el profesorado añoraron las clases presenciales.

Finalmente, la narrativa de las y los especialistas hizo alusión recurrente al hecho de que la operación del Consejo Nacional de Fomento Educativo (CONAFE), así como sus materiales edu-

Diálo@os sobre Educación año 12 | número 22 | enero-junio 2021 | ISSN 2007-2171 
cativos (en particular, los cuadernillos del CONAFE), tuvieron un modesto protagonismo educativo durante la contingencia sanitaria.

F) Agobios: los principales problemas familiares durante la contingencia sanitaria por COVID-19. La pandemia provocó que la casa, la escuela y el trabajo se fusionaran en un mismo espacio. Esta situación, por sí misma fue agobiante, pero se intensificó por varios motivos relacionados con la enseñanza remota de emergencia. En primer lugar, no todas las casas contaban con espacios ideales para el estudio y el trabajo remoto o, por lo menos, no todas estaban acondicionadas para eso. En muchas familias no había suficientes computadoras como para que todos los miembros estudiaran y trabajaran simultáneamente, ni la potencia de internet adecuada para tal fin. Este acondicionamiento repentino supuso poner en juego habilidades de conciliación de horarios, intereses económicos y necesidades familiares. En el caso de las familias que padecían violencia intrafamiliar, esta situación intensificó sus efectos perniciosos.

Las desigualdades entre los miembros de las familias, sobre todo las desigualdades de género, tuvieron efectos diferenciados. Las tareas domésticas, que generalmente eran asumidas por ellas, debieron negociarse porque el confinamiento exigía realizar más limpieza y más orden. Pero, además, porque también ellas se hicieron cargo, en la mayoría de los casos, del acompañamiento de las clases síncronas de las y los estudiantes de la casa. Por extensión, ellas tuvieron que lidiar con la incertidumbre sobre la manera en que las y los alumnos serían evaluados. La falta de retroalimentación oportuna por parte de profesores provocó ansiedad sobre esas evaluaciones. Hubo casos en que los parientes hicieron las tareas, ya sea porque las instrucciones no eran claras o porque eran demasiadas tareas. Por exceso o por inadecuación, la evaluación sumativa fue una simulación.

Las disputas y negociaciones sobre el uso de los dispositivos provocaron que el teléfono inteligente, y en particular, la aplicación WhatsApp, se usaran con fines educativos. De hecho, varias aplicaciones móviles y varias redes sociales fueron usadas con fines educativos. La improvisación ganó protagonismo y quizá favoreció la creatividad, pero no existen evidencias de que haya favorecido el aprendizaje.

La falta de objetos digitales de aprendizaje provocó dos situaciones educativas deseables. En primer lugar, las familias tuvieron que recurrir a expresiones culturales y artísticas locales o en línea para apoyar el aprendizaje. De esta manera se reivindicó un tema postergado en la educación: el aprendizaje de contenidos formales con el apoyo del arte y la cultura locales. En segundo lugar, la familia tuvo que inventar procesos de aprendizaje colaborativo entre sus miembros. De esta manera, las y los alumnos tuvieron que aprender a aprender entre miembros de la familia y no entre pares, como suelen hacerlo en la escuela.

En un principio, el alumnado estuvo ajeno a las decisiones que las autoridades educativas tomaron sobre la enseñanza remota de emergencia, pero paulatinamente fueron tomados en cuenta. Sin embargo, debido a que no existen políticas públicas educativas que incentiven la 
participación vigorosa de las familias en el aprendizaje de contenidos formales, la atención que recibió el alumnado se redujo a reconocer que la enseñanza remota de emergencia no ofreció procesos de socialización, tan necesarios en los procesos educativos como la convivencia entre pares; tampoco ofreció ritos de paso, tan importantes en los procesos simbólicos e identitarios como las titulaciones.

G) Recusamientos: los principales problemas educativos después de la contingencia sanitaria por COVID-19. De acuerdo con las y los especialistas, la contingencia sanitaria dejó patente que la utopía digital no es tal. Ya no es útil continuar con el discurso simplista y dicotómico que coloca a la educación presencial como una opción conservadora, inercial y anticuada. Esto, porque su contraparte, es decir, las modalidades educativas que incorporan tecnología digital no tuvieron un papel innovador ni liberador durante la contingencia sanitaria. Al contrario, sumieron en el desconcierto a la mayoría y subrayó una serie de deficiencias previas. Actualmente, la narrativa sobre la utopía digital podría ser conveniente para unos pero discriminatoria para la mayoría, lo que provoca recursamientos al respecto.

Si bien las actividades docentes desarrolladas durante la contingencia sanitaria no pueden ser consideradas educación virtual ni educación a distancia, lo cierto es que en el imaginario social sí se considera que el SEN se vio obligado a migrar de la modalidad presencial a otra modalidad alternativa. Esta confusión es delicada porque no es deseable que la docencia remota de emergencia sea el referente principal para diseñar planes de continuidad académica. Y no es deseable porque, como ya se ha dicho antes en este texto, sumió en el desconcierto a la mayoría y profundizó las brechas de desigualdad. De tal manera que una lección aprendida refiere a que las contingencias (de cualquier tipo) le suponen a la educación desafíos adaptativos y desafíos técnicos. Evidencia de lo anterior es que hubo muchas reacciones espontáneas y creativas, pero, a falta de planes de continuidad académica, terminaron siendo desmotivadoras. En este sentido, la innovación educativa no solo está relacionada con el uso de la tecnología, sino también con la adaptación y la continuidad en escenarios educativos adversos.

Tampoco la educación a distancia ni la educación virtual o en línea son, conceptualmente, los mejores referentes para rediseñar una modalidad educativa ejecutable durante periodos de contingencia. La educación a distancia es eminentemente institucional porque alude a que en el proceso educativo hay un centro emisor oficial y varios nodos periféricos receptores; a eso refiere el concepto a distancia. Por su parte, la educación virtual o en línea refiere a la virtualización de los objetos y procesos de aprendizaje; por eso, conceptualmente, estaría más cercana al home schooling, home learning o al entorno personal de aprendizaje. En este caso, hay una disrupción entre institución educativa y el aprendiz. Un término emergente que conceptualmente ayudaría a diseñar planes de continuidad académica es la intermodalidad educativa. Esta alude al diseño de procesos educativos que permiten transitar versátilmente entre modalidades institucionales y no institucionales con el objetivo de lograr aprendizajes en cualquier situa- 
ción; por supuesto, también en situaciones contingentes. La intermodalidad educativa supera la narrativa utópica y acrítica sobre la digitalización de los objetos y los procesos educativos porque, como lo mencionaron reiteradamente las y los especialistas, considera seriamente retomar tanto herramientas predigitales como el uso de herramientas digitales. Un ejemplo de lo anterior es considerar a la radio, la televisión y los impresos en los procesos educativos durante contingencias.

Respecto a la paulatina vuelta a la nueva normalidad, la narrativa de las y los especialistas coincidió en que el término normalidad tiene sentido en la estadística, pero en los procesos educativos y sociales es excluyente y discriminatorio. Sería deseable erradicar el término normalidad porque si no se realizan cambios profundos en el SEN, solo volveremos paulatinamente a una normalidad en crisis crónica, que es tal porque no es sensible a la diversidad educativa. Sin embargo, los cambios profundos pueden empezar, ciertamente, con el diseño de planes de continuidad académica. La narrativa de los especialistas coincide en que existe mucho conocimiento científico acumulado que ayudaría a tomar decisiones sobre políticas públicas educativas basadas en evidencia empírica. Es importante que en estas decisiones participen las y los profesores, el alumnado y las familias, y seguir las siguientes ideas sintéticas: a) La tutoría virtual pudo haber solucionado muchos problemas durante la enseñanza remota de emergencia porque no pone el acento en la enseñanza de contenidos formales, sino en la comprensión de los problemas afectivos, familiares, personales que afectan el aprendizaje; $b$ ) Los programas de alfabetización digital institucionales suelen impartirse en situaciones abstractas, pero a partir de ahora podrían diseñarse encaminados al uso de aplicaciones digitales propias de un plan de continuidad académica; c) Sería útil decidir qué contenidos de aprendizaje son preponderantes y cómo serán evaluados durante una contingencia. Sobre ese aspecto advirtieron que, para ser justos, si durante una contingencia no se aclaran y aplican criterios de evaluación a la docencia, tampoco debería haber evaluación al aprendizaje; d) La evaluación no es una medida punitiva, sino un proceso mediador entre aprendizajes previos y nuevos; da fe de que el o la estudiante ha aprendido, pero durante la contingencia lo único que cambia es cómo se hace la evaluación porque el profesor desaparece como mediador y la familia y la conectividad ganan protagonismo al respecto.

Durante la contingencia hubo muchos procesos de horizontalidad entre cuerpos colegiados porque las videoconferencias reunieron a quienes difícilmente se reunirían presencialmente; si se mantiene esta situación, sería posible consolidar liderazgos pedagógicos, que son necesario para impulsar el proceso de diseño de planes de continuidad académica. Finalmente, la narrativa de las y los especialistas coincidió en que crecerá la demanda de matriculación en las universidades públicas porque la crisis económica postCOVID restringirá el acceso a la educación privada. A ese problema habrá que sumarle que nada garantiza que el nuevo ciclo escolar inicie de manera presencial, o sea, que será necesario atender regularizaciones, deserciones y nuevos ingresos por vía virtual. 
H) (Des)involucramientos: los principales problemas familiares después de la contingencia sanitaria por COVID-19. La enseñanza remota de emergencia obligó a las familias a involucrarse en la solución de varios problemas propiamente educativos. Entre los más obvios, se encontraron los problemas de que los parientes, ni son necesariamente educadores formales ni suelen participar activamente en la educación formal del alumnado. Si no se toman las medidas necesarias, después de la contingencia sanitaria podría volver ese distanciamiento entre las familias y la educación formal.

Aunque el desconcierto y las privaciones que caracterizaron a la enseñanza remota de emergencia produjeron ciertas soluciones creativas, en términos generales, ni el alumnado ni las familias desean continuar con procesos educativos remotos. Esta desafección tiene varios motivos, pero destacaron los siguientes: si permanece el alumnado mucho tiempo en casa, tiene que realizar más tareas domésticas; el alumnado acude a las escuelas, también para socializar y convivir con sus pares; en el caso de las y los alumnos que padecen violencia intrafamiliar, asistir a clases presenciales es, incluso, saludable.

Durante la enseñanza remota de emergencia, las familias fueron capaces de desarrollar procesos de aprendizaje alternativos a la burocracia institucional. Por ejemplo, no aplicaron dogmáticamente ni la sistematización ni la dosificación institucional de los contenidos de aprendizaje; no aplicaron métodos de aprendizaje escolarizados y, seguramente, supieron priorizar contenidos relevantes y desestimar contenidos accesorios. Como esos procesos de aprendizaje alternativos surgieron en un espacio íntimo, no existen registros al respecto, solo anécdotas. Esta oportunidad para analizar la metacognición de ese tipo de educación y ampliar la base científica de la educación para toda la vida podría perderse.

\section{Conclusiones}

La suspensión de clases presenciales debido a la pandemia por COVID-19 no implicó el desarrollo de educación a distancia o virtual, sino solamente a la enseñanza remota de emergencia. Este carácter emergente obligó a las familias a lidiar con varios problemas propiamente educativos. Sin embargo, ninguno de esos problemas fue nuevo, sino que forman parte de una serie de desigualdades sociales estructurales y deficiencias del Sistema Educativo Nacional mexicano.

Los resultados de esta metasíntesis sobre la narrativa educativa durante la contingencia sanitaria sugieren que el criterio más adecuado para el análisis al respecto es cronológico: antes, al inicio, durante y después de la contingencia sanitaria. Asimismo, se identificó que dos espacios simbólicos se mantuvieron en tensión en ese periodo de tiempo: las instituciones educativas y las familias. Este criterio y esos espacios simbólicos permitieron distinguir que, el hecho de que la casa, la escuela y el trabajo se hubieran fusionado en un mismo espacio abruptamente, puso en juego todas las capacidades que tienen las familias para minimizar las desigualdades educativas, reorganizarse rápidamente y superar el agobio de lidiar con la burocracia educativa 
institucionalizada. No obstante, debido a que no existen políticas públicas que fomenten la participación de las familias en la educación formal, se prevé que, paulatinamente, volverán a distanciarse del proceso educativo.

En este periodo de contingencia, las familias supieron resolver varios problemas educativos, entre los que destacan, la invención de procesos, métodos y objetos de aprendizaje no institucionalizados. Desafortunadamente, debido a que eso sucedió de manera íntima, entre los miembros de la familia, no han sido propiamente registrados para aumentar la base científica sobre modelos alternativos de aprendizaje. El desafío para la investigación educativa radica en obtener esas anécdotas y convertirlas en conocimiento científico para apoyar la toma de decisiones de las autoridades educativas ante nuevas contingencias.

Desde el punto de vista conceptual, fue patente que la contingencia sanitaria señaló con claridad que ya es momento de trascender la utopía digital, donde se aborda de manera simplista y dicotómica el papel de la educación predigital y digital. La enseñanza remota de emergencia, desarrollada en el periodo de la contingencia, permitió elucidar que no se trata de contraponer la educación a distancia o virtual y la presencial, sino de crear sistemas intermodales, que admitan la combinación de todas las modalidades educativas disponibles para que el alumnado aprenda en cualquier situación; por supuesto, también en situación de contingencia.

Una vez que inicie la reincorporación a la educación habitual, la comunidad educativa podrá hacer muy poco sobre las desigualdades sociales estructurales. Sin embargo, sí puede exigir que las autoridades educativas diseñen planes de continuidad académica basados en evidencia empírica para enfrentar adecuadamente las contingencias que ocurrirán en el futuro, que invariablemente ocurrirán.

\section{Referencias}

ANUIES (2020a). Acciones ante la contingencia y la continuidad de las universidades. https://recursosdigitales.anuies.mx/acciones-de-contingencia-y-continuidad-de-las-universidades/ ANUIES (2020b). ANUIES-TIC. https://anuies-tic.anuies.mx/web/

ANUIES (2020c). ANUIES. http://www.anuies.mx/

ANUIES; ANUIES-TIC; RedLaTE; CUDI (2020). Cómo prepararse ante contingencias en instituciones educativas. https://recursosdigitales.anuies.mx/seminario-como-prepararse-ante-contingencias-en-instituciones-educativas/

Benemérita Universidad Autónoma de Puebla (2020). Continuidad académica. https://dcytic. buap.mx/continuidad-academica/

Breckenridge, J.; D. Jones (2009). Demystifying Theoretical Sampling in Grounded Theory Research. Grounded Theory Review, 8(2). http://groundedtheoryreview.com/2009/06/30/847/ 
Butler, A. E.; B. Copnell; H. Hall (2018). The Development of Theoretical Sampling in Practice. Collegian, 1-6. https://doi.org/10.1016/j.colegn.2018.01.002

Centro de Enseñanza Técnica y Superior (2020). Sobre el COVID-19. https://www.cetys.mx/alertascoronavirus/

COMIE (2020a). COMIE. http://www.comie.org.mx/

COMIE(2020b).Forosvirtuales COMIE.https://www.youtube.com/playlist?list=PL GnpY8IKOzGOSDaKRN7RkZuEAab733LE

CUDI (2020). CUDI. http://www.cudi.edu.mx/eventos/atlas-del-COVID-19

El Colegio de México (2020). Información sobre COVID-19. https://www.colmex.mx/es/informacion-COVID19

Finfgeld-Connett, D. (2010). Generalizability and Transferability of Meta-synthesis Research findings. Journal ofAdvanced Nursing, 66(2), 246-254. https://doi.org/doi:10.1111/j.13652648.2009.05250.x

Finfgeld-Connett, D. (2016). The Future of Theory-generating Meta-synthesis Research. Qualitative Health Research, 26(3), 291-293.

Finfgeld-Connett, D. (2018). A Guide to Qualitative Meta-synthesis. Taylor \& Francis.

Finlayson, K.; A. Dixon (2008). Qualitative Meta-synthesis: A Guide for the Novice. Nurse Researcher, 15(2), 59-71.

Flick, U. (2015). Doing Grounded Theory (U. Flick (ed.); 2a ed.). SAGE Publications.

Gobierno de México (2020). Sana distancia COVID- 19. https://www.gob.mx/salud/documentos/ sana-distancia

Guetterman, T. C.;W. A. Babchuk; M. C. Howell Smith; J. Stevens (2017). Contemporary Approaches to Mixed Methods-Grounded Theory Research: A Field-Based Analysis. Journal of Mixed Methods Research, 1(17), 1-17. https://doi.org/10.1177/1558689817710877

Instituto Politécnico Nacional (2020). Plan de Continuidad Académica en el IPN. https://elementosdeaprendizaje.jpn.mx

Leary, H.; A. Walker (2018). Meta-Analysis and Meta-Synthesis Methodologies: Rigorously Piecing Together Research. TechTrends. https://doi.org/10.1007/s11528-018-0312-7

RedLaTE. (2020). RedLaTE. https://redlate.net/

SEP (2020). SIGED Sistema de Información y Gestión Educativa. https://www.siged.sep.gob.mx/SIGED/principalesCifras.html

Tecnológico de Monterrey (2020a). COVID-19 Recursos educativos: Observatorio de Innovación Educativa. https://observatorio.tec.mx/COVID19-recursos-educativos

Tecnológico de Monterrey (2020b). Transforma tu práctica docente para la continuidad académica. https://innovacioneducativa.tec.mx/continuidad-academica/

Tecnológico Superior de Irapuato (2020). Plataforma de Educación a Distancia. https://ead.itesi. edu.mx/ 
UNESCO (2020a). 1.370 millones de estudiantes ya están en casa con el cierre de las escuelas de COVID-19. https://es.unesco.org/news/1370-millones-estudiantes-ya-estan-casa-cierre-escuelas-COVID-19-ministros-amplian-enfoques

UNESCO (2020b). Impacto de COVID en la educación. https://es.unesco.org/COVID19/educationresponse

Universidad Anáhuac (2020). Coronavirus COVID 19. https://www.anahuac.mx/mexico/COVID-19 Universidad Autónoma de Baja California (2020). UABC Informa: Coronavirus. http://gaceta.uabc. $\mathrm{mx} /$ coronavirus/

Universidad Autónoma de Ciudad Juárez (2020). Programa de Continuidad Académica Virtual. http://www3.uacj.mx/CSB/BIVIR/Paginas/BD open.aspx

Universidad Autónoma de Guerrero (2020a). Plan de Contingencia Académica utilizando TIC. http://virtual.uagro.mx/plancontingencia.pdf

Universidad Autónoma de Guerrero (2020b). Plan de Continuidad Académica UAGro en Línea. http://virtual.uagro.mx/plancontinuidad/documentos/ssss.pdf

Universidad Autónoma de Nuevo León (2020). Estrategia Digital para Contingencia COVID-19. https://www.uanl.mx/COVID-19/

Universidad Autónoma del Estado de Hidalgo (2020). Acciones importantes. https://www.uaeh. edu.mx/COVID-19/

Universidad Autónoma del Estado de México (2020a). Aula FACICO. https://www.facico-uaemex. $\underline{\mathrm{mx} / 2018-2022 / \text { aulafacico.html }}$

Universidad Autónoma del Estado de México (2020b). Clases Virtuales ante contingencia. https:// campusvirtual.uaemex.mx/contingencia/index.php

Universidad Autónoma del Estado de México (2020c). Clases virtuales ante la contingencia sanitaria. https://revado.uaemex.mx/avisos/Clases virtuales ante la contingencia sanitaria. pdf

Universidad Autónoma del Estado de México (2020d). Dirección de Educación Continua y a Distancia. https://www.seduca.uaemex.mx/

Universidad Autónoma del Estado de México (2020e). Herramientas TIC. http://tics.uaemex.mx/ Universidad Autónoma del Estado de Morelos (2020). UAEM-VA: Protocolo de continuidad virtual del aprendizaje. http://portal.e-uaem.mx/uaem-va/

Universidad Autónoma Metropolitana-Azcapotzalco (2020a). Contingencia COVID-19. http:// contingencia.azc.uam.mx/

Universidad Autónoma Metropolitana-Azcapotzalco (2020b). Tutoriales. Contingencia COVID 19. http://contingencia.azc.uam.mx/tutoriales.php

Universidad Cristóbal Colón (2020). Continuidad académica UCC en caso de contingencia. https:// sites.google.com/ucc.mx/docencia-y-contingencia/ 
Universidad de Ciencias y Artes de Chiapas (2020a). Plan de contingencia. https://contingencia. unicach.mx/

Universidad de Ciencias y Artes de Chiapas (2020b). Plan de Continuidad Académica UNICACH. https://contingencia.unicach.mx/

Universidad de Colima (2020). Universidad de Colima ante el COVID-19. https://portal.ucol.mx/ COVID-19/

Universidad de Guanajuato (2020a). Programa EduTIC UG. https://edutics.ugto.mx/

Universidad de Guanajuato (2020b). UG en tu casa. https://edutics.ugto.mx/

Universidad de las Américas Puebla (2020a). Campañas de salud mental durante el autoaislamiento. https://www.udlap.mx/COVID19/cuidate.aspx - saludFisica

Universidad de las Américas Puebla (2020b). Implantación de medidas sanitarias en sitio. https:// www.udlap.mx/COVID19/informate-y-previene.aspx

Universidad de las Américas Puebla (2020c). Respuesta de la Universidad de las Américas Puebla ante el COVID-19 (Coronavirus). https://www.udlap.mx/COVID19/

Universidad de Sonora (2020). Plan de continuidad académica-docencia por contingencia COVID-19. http://www.continuidadacademica.unison.mx/

Universidad del Caribe (2020). Herrarmientas digitales. https://www.unicaribe.mx/files/COVID-19/avisos-COVID-19-biblioteca.pdf

Universidad del Valle de México (2020). ¿Tienes duda sobre las clases virtuales y el uso de la plataforma teams? https://landing.uvm.mx/intro-teams/

Universidad Ibero CDMX (2020a). IBERO COVID-19. https://contingencia-COVID.ibero.mX/

Universidad Ibero CDMX (2020b). Lleva tu curso a la red. https://sites.google.com/view/tucursovirtual//

Universidad La Salle (2020). COVID-19 Disposiciones Oficiales. https://lasalle.mx/COVID/

Universidad Nacional Autónoma de México (2020a). ¿Cómo puedo continuar mi curso ante una contingencia? https://www.codeic.unam.mx/index.php/contingencia cursos/

Universidad Nacional Autónoma de México (2020b). Campus Virtual CUAED. https://distancia. cuaed.unam.mx/campusvirtual.html

Universidad Panamericana (2020). Tu Salud es Primero. https://universidadpanamericanaCOVID19.com/

Universidad Popular Autónoma del Estado de Puebla (2020). UPAEP sigue adelante. https:// upaep.mx/plandecontinuidad/

Universidad Regiomontana (2020). Informes sobre COVID-19. https://www.COVID19.u-erre.mx/

Universidad Tecnológica de Jalisco (2020). Contingencia COVID-19. https://plai.mx/

Universidad Tecnológica Tula-Tepeji (2020). Mi universidad en casa. http://www.uttt.edu.mx/ miuniversidadencasa/

Universidad Veracruzana (2020). Plan de contingencia COVID 19. https://www.uv.mx/plandecontingencia/ 


\section{Anexo 1}

Seminario "Cómo prepararse ante contingencias en instituciones educativas" de ANUIES, CUDI y RedLaTE

\begin{tabular}{|c|c|c|c|}
\hline Región & Universidad/Institución & Acciones & Síntesis \\
\hline \multirow[t]{5}{*}{$\begin{array}{l}\text { Región Sur- } \\
\text { Sureste }\end{array}$} & $\begin{array}{l}\text { Universidad Veracruzana } \\
(2020)\end{array}$ & $\begin{array}{l}\text { 1. Plan de contin- } \\
\text { gencia COVID-19 }\end{array}$ & $\begin{array}{l}\text { Presenta planes de acción durante la } \\
\text { contingencia, comunicados oficiales } \\
\text { de la universidad y una campaña infor- } \\
\text { mativa. }\end{array}$ \\
\hline & $\begin{array}{l}\text { Universidad del Caribe } \\
(2020)\end{array}$ & $\begin{array}{l}\text { 2. Herramientas } \\
\text { digitales }\end{array}$ & $\begin{array}{l}\text { Presenta cinco buscadores de literatu- } \\
\text { ra científica. }\end{array}$ \\
\hline & $\begin{array}{l}\text { Universidad Autónoma } \\
\text { deYucatán }\end{array}$ & $\begin{array}{l}\text { 3. Información so- } \\
\text { bre COVID-19 }\end{array}$ & $\begin{array}{l}\text { Presenta información médica sobre el } \\
\text { COVID-19. }\end{array}$ \\
\hline & \multirow[t]{2}{*}{$\begin{array}{l}\text { Universidad de Ciencias y } \\
\text { Artes de Chiapas (2020b, } \\
\text { 2020a) }\end{array}$} & $\begin{array}{l}\text { 4. Plan de Conti- } \\
\text { nuidad Académica } \\
\text { UNICACH }\end{array}$ & $\begin{array}{l}\text { Presenta cinco opciones de capacita- } \\
\text { ción sobre educación en línea (acceso } \\
\text { limitado a la comunidad de la UNI- } \\
\text { CACH). }\end{array}$ \\
\hline & & $\begin{array}{l}\text { 5. Plan de Contin- } \\
\text { gencia }\end{array}$ & $\begin{array}{l}\text { Acceso limitado a la comunidad de la } \\
\text { UNICACH }\end{array}$ \\
\hline & $\begin{array}{l}\text { Universidad Cristóbal } \\
\text { Colón (2020) }\end{array}$ & $\begin{array}{l}\text { 6. Continuidad } \\
\text { académica UCC en } \\
\text { caso de contingen- } \\
\text { cia }\end{array}$ & $\begin{array}{l}\text { Presenta un micrositio con sugeren- } \\
\text { cias frente a contingencias en diver- } \\
\text { sos ámbitos, como la comunicación, } \\
\text { formatos, agendas de estudio y di- } \\
\text { rectrices para evaluación durante la } \\
\text { contingencia, además de varios links } \\
\text { a recursos en línea y a sitios de otros } \\
\text { organismos nacionales que abordan el } \\
\text { problema de la falta de planes de con- } \\
\text { tinuidad académica }\end{array}$ \\
\hline \multirow[t]{4}{*}{$\begin{array}{l}\text { Región Centro- } \\
\text { Sur }\end{array}$} & \multirow[t]{2}{*}{$\begin{array}{l}\text { Universidad Autónoma } \\
\text { de Guerrero (2020b, } \\
\text { 2020a) }\end{array}$} & $\begin{array}{l}\text { 7. Plan de Conti- } \\
\text { nuidad Académica } \\
\text { UAGro en Línea }\end{array}$ & $\begin{array}{l}\text { Presenta propuestas de capacitación a } \\
\text { docentes, alumnado, plan de gestión } \\
\text { de crisis con estrategias reactivas, } \\
\text { proactivas y accesibles, así como algu- } \\
\text { nas acciones y responsables institucio- } \\
\text { nales. }\end{array}$ \\
\hline & & $\begin{array}{l}\text { 8. Plan de Contin- } \\
\text { gencia Académica } \\
\text { utilizando TIC }\end{array}$ & $\begin{array}{l}\text { Presenta un plan de contingencia con } \\
\text { cinco fases, un plan de gestión de cri- } \\
\text { sis y un plan operativo de monitoreo. }\end{array}$ \\
\hline & $\begin{array}{l}\text { Universidad Tecnológica } \\
\text { de Tula-Tepeji (2020) }\end{array}$ & $\begin{array}{l}\text { 9. Mi universidad } \\
\text { en casa }\end{array}$ & $\begin{array}{l}\text { Presenta el acceso a las aulas virtuales } \\
\text { de los programas de grado y posgra- } \\
\text { do, así como links de sitios de cultura. }\end{array}$ \\
\hline & $\begin{array}{l}\text { Universidad de las Améri- } \\
\text { cas Puebla (2020C, 2020a, } \\
2020 b)\end{array}$ & $\begin{array}{l}\text { 10. Respuesta de la } \\
\text { Universidad de las } \\
\text { Américas Puebla } \\
\text { ante el COVID-19 } \\
\text { (Coronavirus) }\end{array}$ & $\begin{array}{l}\text { Presenta protocolos de acción para } \\
\text { enseñar, aprender y trabajar en línea, } \\
\text { comunicados oficiales del rector y su- } \\
\text { gerencias de prevención. }\end{array}$ \\
\hline
\end{tabular}




\begin{tabular}{|c|c|c|c|}
\hline & $\begin{array}{l}\text { Universidad Popular Au- } \\
\text { tónoma del Estado de } \\
\text { Puebla (2020) }\end{array}$ & $\begin{array}{l}\text { 11. UPAEP sigue } \\
\text { adelante }\end{array}$ & $\begin{array}{l}\text { Micrositio dirigido a docentes, alum- } \\
\text { nos, administrativos, familiares y } \\
\text { aspirantes, que presenta recursos tec- } \\
\text { nológicos disponibles para acompañar } \\
\text { el proceso de migración, los avisos y } \\
\text { comunicaciones oficiales emanados } \\
\text { por las diversas áreas, las últimas no- } \\
\text { ticias sobre el COVID-19 y una serie de } \\
\text { recomendaciones higiénico-sanitarias. }\end{array}$ \\
\hline & $\begin{array}{l}\text { Universidad Autónoma } \\
\text { del Estado de Morelos } \\
\text { (2020) }\end{array}$ & $\begin{array}{l}\text { 12. UAEM-VA: Pro- } \\
\text { tocolo de conti- } \\
\text { nuidad virtual del } \\
\text { aprendizaje }\end{array}$ & $\begin{array}{l}\text { Presenta el protocolo para crear aulas } \\
\text { virtuales de manera expedita y las } \\
\text { vías para capacitarse en su uso. La } \\
\text { información está dirigida a docentes y } \\
\text { alumnado. }\end{array}$ \\
\hline & \multirow[t]{5}{*}{$\begin{array}{l}\text { Universidad Autónoma } \\
\text { del Estado de México } \\
\text { (2020b, 2020d, 2020e, } \\
\text { 2020c, 2020a) }\end{array}$} & $\begin{array}{l}\text { 13. Clases virtuales } \\
\text { ante contingencia }\end{array}$ & $\begin{array}{l}\text { Presenta información sobre el eco- } \\
\text { sistema de educación virtual y mixta: } \\
\text { Moodle, Microsoft Teams y SEDUCA. } \\
\text { Dirigido a docentes. }\end{array}$ \\
\hline & & $\begin{array}{l}\text { 14. Dirección de } \\
\text { Educación Conti- } \\
\text { nua y a Distancia }\end{array}$ & $\begin{array}{l}\text { Presenta la página oficial de la Direc- } \\
\text { ción de Educación Continua y a Dis- } \\
\text { tancia. }\end{array}$ \\
\hline & & $\begin{array}{l}\text { 15. Herramientas } \\
\text { TIC }\end{array}$ & $\begin{array}{l}\text { Presenta el ingreso a Moodle, Micro- } \\
\text { soft Teams, SEDUCA y G Suite Educa- } \\
\text { tion. Acceso limitado con usuario y } \\
\text { contraseña. }\end{array}$ \\
\hline & & $\begin{array}{l}\text { 16. Clases virtuales } \\
\text { ante la contingen- } \\
\text { cia sanitaria }\end{array}$ & $\begin{array}{l}\text { Presenta acciones didácticas para sus- } \\
\text { tituir las clases presenciales con aplica- } \\
\text { ciones de tecnología digital }\end{array}$ \\
\hline & & 17. Aula FACICO & $\begin{array}{l}\text { Presenta información sobre aplicacio- } \\
\text { nes digitales, recursos web y MOOC. }\end{array}$ \\
\hline & $\begin{array}{l}\text { Universidad Autónoma } \\
\text { del Estado de Hidalgo } \\
\text { (2020) }\end{array}$ & $\begin{array}{l}\text { 18. Acciones im- } \\
\text { portantes }\end{array}$ & $\begin{array}{l}\text { Presenta acceso a su plataforma ins- } \\
\text { titucional y a la biblioteca digital con } \\
\text { acceso limitado por usuario y contra- } \\
\text { seña. Además, pone a disposición el } \\
\text { repositorio institucional e información } \\
\text { médica sobre COVID-19. }\end{array}$ \\
\hline & $\begin{array}{l}\text { Benemérita Universidad } \\
\text { Autónoma de Puebla } \\
\text { (2020) }\end{array}$ & $\begin{array}{l}\text { 19. Continuidad } \\
\text { académica }\end{array}$ & $\begin{array}{l}\text { Presenta tutoriales para Microsoft } \\
\text { Teams y G Suite Education, entre otros } \\
\text { recursos en línea. }\end{array}$ \\
\hline $\begin{array}{l}\text { Región Metro- } \\
\text { politana }\end{array}$ & $\begin{array}{l}\text { El Colegio de México A.C. } \\
(2020)\end{array}$ & $\begin{array}{l}\text { 20. Información } \\
\text { sobre COVID-19 }\end{array}$ & $\begin{array}{l}\text { Presenta información sobre el CO- } \\
\text { VID-19, acceso al Moodle institucional, } \\
\text { los servicios de la biblioteca, recursos } \\
\text { de información en acceso abierto, in- } \\
\text { formación sobre las clases remotas. }\end{array}$ \\
\hline
\end{tabular}




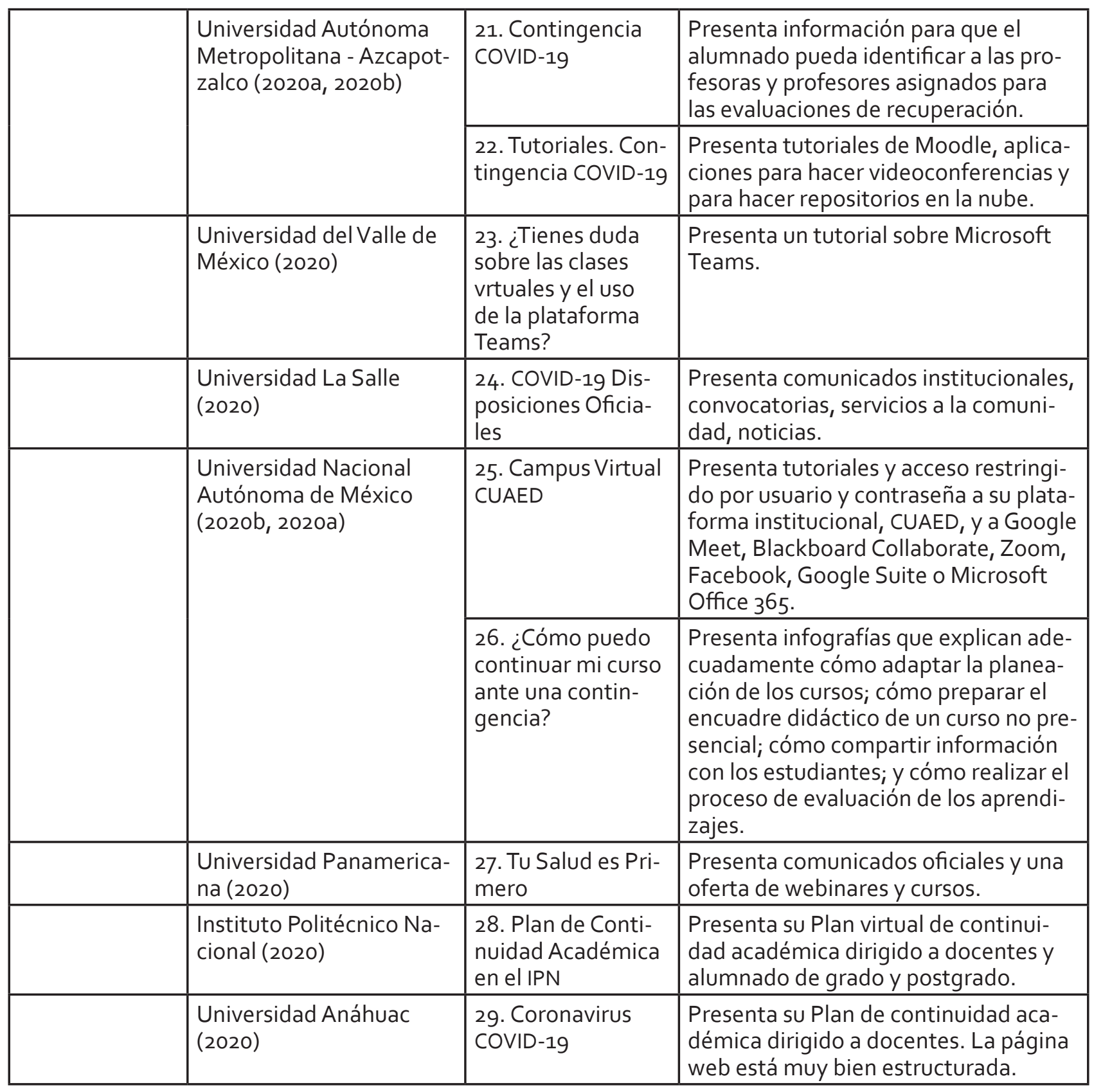




\begin{tabular}{|c|c|c|c|}
\hline & \multirow[t]{2}{*}{$\begin{array}{l}\text { Universidad Ibero CDMX } \\
\text { (2020b, 2020a) }\end{array}$} & $\begin{array}{l}\text { 30. Lleva tu curso a } \\
\text { la red }\end{array}$ & $\begin{array}{l}\text { Presenta de manera ordenada infor- } \\
\text { mación y recursos para crear cursos } \\
\text { virtuales: una guía sintética, procesos } \\
\text { de digitalización, modelos de cursos, } \\
\text { metodologías didácticas. para la vir- } \\
\text { tualidad, herramientas para digitalizar, } \\
\text { y criterios para evaluar los cursos vir- } \\
\text { tuales. }\end{array}$ \\
\hline & & 31. IBERO COVID-19 & $\begin{array}{l}\text { Presenta información médica, comu- } \\
\text { nicados, noticias, pero también una } \\
\text { guía dirigida a profesoras y profesores } \\
\text { para el cierre del semestre a distancia. } \\
\text { Esta guía contiene varios temas senci- } \\
\text { llos y útiles. }\end{array}$ \\
\hline \multirow[t]{4}{*}{ Región Noreste } & \multirow[t]{2}{*}{$\begin{array}{l}\text { Tecnológico de Monte- } \\
\text { rrey (2020b, 2020a) }\end{array}$} & $\begin{array}{l}\text { 32. Transforma tu } \\
\text { práctica docente } \\
\text { para la continuidad } \\
\text { académica }\end{array}$ & $\begin{array}{l}\text { Presenta una guía muy completa y } \\
\text { ordenada para migrar cursos a la edu- } \\
\text { cación virtual. Contiene formatos y } \\
\text { recursos. }\end{array}$ \\
\hline & & $\begin{array}{l}\text { 33. COVID-19 Re- } \\
\text { cursos educativos: } \\
\text { Observatorio de } \\
\text { Innovación Educa- } \\
\text { tiva }\end{array}$ & $\begin{array}{l}\text { Presenta una compilación nutrida de } \\
\text { webinares, recursos para docentes, } \\
\text { para el alumnado y personal no docen- } \\
\text { te frente a la contingencia sanitaria. } \\
\text { Todo lo anterior dentro de su Observa- } \\
\text { torio de Innovación Educativa. }\end{array}$ \\
\hline & $\begin{array}{l}\text { Universidad Autónoma } \\
\text { de Nuevo León (2020) }\end{array}$ & $\begin{array}{l}\text { 34. Estrategia Digi- } \\
\text { tal para Contingen- } \\
\text { cia COVID-19 }\end{array}$ & $\begin{array}{l}\text { Presenta guías para docentes y alum- } \\
\text { nado para adaptar los procesos de } \\
\text { enseñanza y aprendizaje presencial a } \\
\text { la modalidad en línea. }\end{array}$ \\
\hline & $\begin{array}{l}\text { Universidad Regiomonta- } \\
\text { na (2020) }\end{array}$ & $\begin{array}{l}\text { 35. Informes sobre } \\
\text { COVID-19 }\end{array}$ & $\begin{array}{l}\text { Presenta comunicados oficiales en } \\
\text { forma de blog. }\end{array}$ \\
\hline $\begin{array}{l}\text { Región Centro- } \\
\text { Occidente }\end{array}$ & $\begin{array}{l}\text { Tecnológico Superior de } \\
\text { Irapuato (2020) }\end{array}$ & $\begin{array}{l}\text { 36. Plataforma de } \\
\text { Educación a Dis- } \\
\text { tancia }\end{array}$ & $\begin{array}{l}\text { Presenta la Plataforma de Educación } \\
\text { a Distancia institucional sin especifica- } \\
\text { ciones para la contingencia sanitaria. }\end{array}$ \\
\hline & \multirow[t]{2}{*}{$\begin{array}{l}\text { Universidad de Guanajua- } \\
\text { to }(2020 b)(2020 a)\end{array}$} & 37. UG en tu casa & $\begin{array}{l}\text { Presenta recursos sobre ciencia, aca- } \\
\text { demia, cultura y recreación virtual. } \\
\text { Dentro de esta oferta presenta un link } \\
\text { a tutoriales para implementar estrate- } \\
\text { gias didácticas digitales. }\end{array}$ \\
\hline & & $\begin{array}{l}\text { 38. Programa Edu- } \\
\text { TIC UG }\end{array}$ & $\begin{array}{l}\text { Presenta la página oficial del progra- } \\
\text { ma institucional eduTIC que contiene } \\
\text { cursos e información sobre Microsoft } \\
\text { Teams, Webex, One Drive, Share Point } \\
\text { y Skype. }\end{array}$ \\
\hline
\end{tabular}




\begin{tabular}{|c|c|c|c|}
\hline & $\begin{array}{l}\text { Universidad de Colima } \\
(2020)\end{array}$ & $\begin{array}{l}\text { 39. Universidad de } \\
\text { Colima ante el CO- } \\
\text { VID-19 }\end{array}$ & $\begin{array}{l}\text { Presenta comunicados, infografías, } \\
\text { recursos digitales y un micrositio titu- } \\
\text { lado Continuidad educativa. En esta } \\
\text { sección hay un PDF detallado sobre su } \\
\text { Programa Universitario de Contingen- } \\
\text { cia ante el COVID-19. }\end{array}$ \\
\hline & $\begin{array}{l}\text { Universidad Tecnológica } \\
\text { de Jalisco (2020) }\end{array}$ & $\begin{array}{l}\text { 40. Contingencia } \\
\text { COVID-19 }\end{array}$ & $\begin{array}{l}\text { Presenta la Plataforma Abierta de In- } \\
\text { novación (PLAi), de Gobierno del Esta- } \\
\text { do de Jalisco operada por la Secretaría } \\
\text { de Innovación Ciencia y Tecnología } \\
\text { (SICyT) del estado de Jalisco. }\end{array}$ \\
\hline \multirow[t]{4}{*}{$\begin{array}{l}\text { Región Noroes- } \\
\text { te }\end{array}$} & $\begin{array}{l}\text { Universidad de Sonora } \\
(2020)\end{array}$ & $\begin{array}{l}\text { 41. Plan de conti- } \\
\text { nuidad académica- } \\
\text { docencia por con- } \\
\text { tingencia COVID-19 }\end{array}$ & $\begin{array}{l}\text { Presenta los recursos institucionales } \\
\text { (Microsoft } 365 \text { y Moodle), buenas } \\
\text { prácticas para continuidad académica, } \\
\text { actividades sencillas y básicas para } \\
\text { asegurar una continuidad académica } \\
\text { durante la contingencia del COVID-19. }\end{array}$ \\
\hline & $\begin{array}{l}\text { Centro de Enseñanza } \\
\text { Técnica y Superior (2020) }\end{array}$ & $\begin{array}{l}\text { 42. Sobre el CO- } \\
\text { VID-19 }\end{array}$ & $\begin{array}{l}\text { Presenta un comunicado mencionan- } \\
\text { do que la institución ha habilitado más } \\
\text { de } 100 \text { cursos y talleres, más de } 33 \\
\text { salas de apoyos virtuales, fortalecido } \\
\text { la infraestructura de docencia remota } \\
\text { y adquirido más de } 850 \text { licencias profe- } \\
\text { sionales de Zoom. }\end{array}$ \\
\hline & $\begin{array}{l}\text { Universidad Autónoma } \\
\text { de Baja California (2020) }\end{array}$ & $\begin{array}{l}\text { 43. UABC Informa: } \\
\text { Coronavirus }\end{array}$ & $\begin{array}{l}\text { Presenta información médica, medi- } \\
\text { das de protección, comunicados ofi- } \\
\text { ciales, avisos y el plan de continuidad } \\
\text { académica. Este contiene información } \\
\text { para docentes y alumnado. }\end{array}$ \\
\hline & $\begin{array}{l}\text { Universidad Autónoma } \\
\text { de Ciudad Juárez (2020) }\end{array}$ & $\begin{array}{l}\text { 44. Programa de } \\
\text { Continuidad Aca- } \\
\text { démica Virtual }\end{array}$ & $\begin{array}{l}\text { Presenta un repositorio de páginas } \\
\text { web relacionadas con recursos digita- } \\
\text { les, pero no existe un plan de continui- } \\
\text { dad académica. }\end{array}$ \\
\hline
\end{tabular}

Fuente: ANUIES (2020a). 


\section{Anexo 2}

Foros virtuales de análisis del COMIE

\begin{tabular}{|c|c|c|}
\hline $\begin{array}{c}\text { Título del foro vir- } \\
\text { tual / duración }\end{array}$ & Fecha & Instituciones participantes \\
\hline $\begin{array}{l}\text { 1. La investigación } \\
\text { educativa en tiempos } \\
\text { del COVID-19 } \\
204 \text { minutos }\end{array}$ & $\begin{array}{l}24 \text { de abril } \\
\text { de } 2020\end{array}$ & $\begin{array}{l}\text { Marcela Gajardo, Facultad Latinoamericana de Ciencias Sociales-Chile; } \\
\text { Judith Kalman, Centro de Investigación y de Estudios Avanzados del } \\
\text { Instituto Politecnico Nacional-Mexico; Cenobio Popoca, Benemérita } \\
\text { Escuela Nacional de Maestros-México; Fernando Reimers, Universidad } \\
\text { de Harvard, miembro de la Comisión sobre Futuros de la Educación de } \\
\text { la Organización de las Naciones Unidas para la Educación, la Ciencia y } \\
\text { la Cultura-Estados Unidos; Sylvia Schmelkes, Universidad' Iberoameri- } \\
\text { cana-México; Rosa María Torres, especialista en educación alternativa- } \\
\text { Ecuador. }\end{array}$ \\
\hline $\begin{array}{l}\text { 2. La educación } \\
\text { básica y los desafios } \\
\text { impuestos por el } \\
\text { COVID-19 y el confi- } \\
\text { namiento sanitario } \\
\quad 127 \text { minutos }\end{array}$ & $\begin{array}{l}14 \text { de mayo } \\
\text { de } 2020\end{array}$ & $\begin{array}{l}\text { Karlena María Cárdenas Espinoza, Facultad de Estudios Superiores } \\
\text { Iztacala, Universidad Nacional Autónoma de México-México; Juan } \\
\text { Carlos Mijangos Noh, Universidad Autónoma de Yucatán-México; Eu- } \\
\text { riel Rosas Lozada, Director de la Escuela Primaria Federal Ford Núm. } \\
\text { 15 José Vasconcelos, Servicios Educativos Integrados al Estado de } \\
\text { México-México; Claudia Santizo Rodall, Universidad Autónoma Me- } \\
\text { tropolitana, Cuajimalpa-México; Saúl Vázquez Rodríguez, Unidad } 201 \\
\text { Oaxaca, Universidad Pedagógica Nacional-México. }\end{array}$ \\
\hline $\begin{array}{l}\text { 3. Los docentes de } \\
\text { educación básica en } \\
\text { México ante el CO- } \\
\text { VID- } 19 \\
\quad 114 \text { minutos }\end{array}$ & $\begin{array}{l}14 \text { de mayo } \\
\text { de } 2020\end{array}$ & $\begin{array}{l}\text { Patricia Ducoing Watty, Universidad Nacional Autónoma de México- } \\
\text { México; Mario Chávez Campos, Dirección General de Educación Su- } \\
\text { perior para Profesionales de la Educación-México; Alberto Ramírez } \\
\text { Martinell, Universidad Veracruzana-México; Rosa María Torres Her- } \\
\text { nández, Universidad Pedagógica Nacional-México; María del Carmen } \\
\text { Gabriela Flores Talavera, Instituto Transdisciplinario de Estudios de la } \\
\text { Consciencia-México; Germán Iván Martínez Gómez, Escuela Normal } \\
\text { Tenancingo-México. }\end{array}$ \\
\hline $\begin{array}{l}\text { 4. La educación } \\
\text { superior ante el CO- } \\
\text { VID-19 y el confina- } \\
\text { miento sanitario en } \\
\text { México } \\
\quad 147 \text { minutos }\end{array}$ & $\begin{array}{l}19 \text { de mayo } \\
\text { de } 2020\end{array}$ & $\begin{array}{l}\text { Adrián Acosta Silva, Universidad de Guadalajara-México; Alejandro } \\
\text { Emanuelle Menéndez, Centro de Lenguas Extranjeras, Unidad Santo } \\
\text { Tomás del Instituto Politécnico Nacional-México; Jesús Francisco Galaz } \\
\text { Fontes, Universidad Autónoma Metropolitana Azcapotzalco-México; } \\
\text { Rosalba Ramírez García, Departamento de Investigaciones Educativas, } \\
\text { Centro de Investigación y de Estudios Avanzados del Instituto Politéc- } \\
\text { nico Nacional-México; Elisa Lugo Villaseñor, Universidad Autónoma } \\
\text { del Estado de México-México. }\end{array}$ \\
\hline $\begin{array}{l}\text { 5. La educación me- } \\
\text { dia superior y el con- } \\
\text { finamiento sanitario } \\
\text { en México } \\
127 \text { minutos }\end{array}$ & $\begin{array}{l}19 \text { de mayo } \\
\text { de } 2020\end{array}$ & $\begin{array}{l}\text { María de Ibarrola Nicolín, Departamento de Investigaciones Educa- } \\
\text { tivas, Centro de Investigación y de Estudios Avanzados del Instituto } \\
\text { Politécnico Nacional-México; Carlota Guzmán Gómez, Universidad } \\
\text { Nacional Autónoma de México-México; Juan Fidel Zorrilla Alcalá, Uni- } \\
\text { versidad Nacional Autónoma de México-México; Sylvia Ortega Salazar, } \\
\text { Consejo Nacional de Educación Profesional Técnica-México; Alfredo } \\
\text { Hualde Alfaro, Colegio de la Frontera Norte-México. }\end{array}$ \\
\hline $\begin{array}{l}\text { 6. La educación su- } \\
\text { perior en la Ciudad } \\
\text { de México, Hidalgo, } \\
\text { Morelos, Puebla, } \\
\text { Tlaxcala y Querétaro } \\
\quad 111 \text { minutos }\end{array}$ & $\begin{array}{l}25 \text { de mayo } \\
\text { de } 2020\end{array}$ & $\begin{array}{l}\text { Gabriela Croda Borges, Universidad Popular Autónoma del Estado de } \\
\text { Puebla-México; Ana Hirsch Adler, Universidad Nacional Autónoma } \\
\text { de México-México; Amelia Molina García, Universidad Autónoma de } \\
\text { Hidalgo-México; Ana Bertha Luna Miranda, Universidad Autónoma de } \\
\text { Tlaxcala-México; Cony Brunhilde Saenger Pedrero, Universidad Autó- } \\
\text { noma del Estado de México-México. }\end{array}$ \\
\hline
\end{tabular}




\begin{tabular}{|c|c|c|}
\hline $\begin{array}{l}\text { 7. Docencia no pre- } \\
\text { sencial de emergen- } \\
\text { cia: lecciones apren- } \\
\text { didas } \\
\quad 109 \text { minutos }\end{array}$ & $\begin{array}{l}26 \text { de mayo } \\
\text { de } 2020\end{array}$ & $\begin{array}{l}\text { Lilian Kravzov Appe, Rectora de la Universidad Abierta y a Distancia de } \\
\text { México-México; Inés Dussel, Centro de Investigación y Estudios Avan- } \\
\text { zados del Instituto Politécnico Nacional-México; Alexandro Escudero } \\
\text { Nahón, Universidad Autónoma de Querétaro-México; Rosario Rogel } \\
\text { Salazar, Universidad Autónoma del Estado de México-México; Germán } \\
\text { Ruiz Méndez, Director de Difusión Digital de Televisión Educativa- } \\
\text { México. }\end{array}$ \\
\hline $\begin{array}{l}\text { 8. La educación en } \\
\text { Oaxaca, Tamaulipas, } \\
\text { Veracruz y Puebla } \\
\text { ante el COVID-19 } \\
\quad 125 \text { minutos }\end{array}$ & $\begin{array}{l}27 \text { de mayo } \\
\text { de } 2020\end{array}$ & $\begin{array}{l}\text { Olga Grijalva Martínez, Universidad Autónoma Benito Juárez de Oa- } \\
\text { xaca-México; Marco Aurelio Navarro Leal, Sociedad Mexicana de Edu- } \\
\text { cación Comparada, UAT-México; Elizabeth Ocampo Gómez, Univer- } \\
\text { sidad Veracruzana-México; Wietse de Vries, Benemérita Universidad } \\
\text { Autónoma de Puebla-México; Ernesto Treviño Ronzón, Universidad } \\
\text { Veracruzana-México. }\end{array}$ \\
\hline $\begin{array}{l}\text { 9. ¿Quién está detrás } \\
\text { de la pantalla? } \\
108 \text { minutos }\end{array}$ & $\begin{array}{l}28 \text { de mayo } \\
\text { de } 2020\end{array}$ & $\begin{array}{l}\text { Juan Carlos Silas Casillas, Instituto Tecnológico y de Estudios Superio- } \\
\text { res de Occidente, Universidad Jesuita de Guadalajara-México; Francis- } \\
\text { co Morfín Otero, Instituto Superior Intercultural Ayuuk-México, Sylvia } \\
\text { Vázquez Rodríguez, Instituto Tecnológico y de Estudios Superiores de } \\
\text { Occidente-México, Mónica López Ramírez, Universidad Nacional Au- } \\
\text { tónoma de México-México. }\end{array}$ \\
\hline $\begin{array}{l}\text { 10. La educación am- } \\
\text { biental en tiempos de } \\
\text { pandemia } \\
\quad 119 \text { minutos }\end{array}$ & $\begin{array}{l}29 \text { de mayo } \\
\text { de } 2020\end{array}$ & $\begin{array}{l}\text { Edgar González Gaudiano, Universidad Veracruzana-México; Elba } \\
\text { Castro Rosales, Universidad de Guadalajara-México; Helio García Cam- } \\
\text { pos, Secretaría de Medio Ambiente y Recursos Naturales-México; Luz } \\
\text { María Nieto Caraveo, Academia Nacional de Educación Ambiental, } \\
\text { A.C.-México; Raúl Cuéllar Ramírez, Ventana Ambiental Desarrollo Sus- } \\
\text { tentable, A. C.-México. }\end{array}$ \\
\hline $\begin{array}{l}\text { 11. Consecuencias } \\
\text { del confinamiento } \\
\text { sanitario en la con- } \\
\text { vivencia, disciplina y } \\
\text { violencia } \\
\quad 115 \text { minutos }\end{array}$ & $\begin{array}{l}1 \text { de junio } \\
\text { de } 2020\end{array}$ & $\begin{array}{l}\text { Cecilia Fierro Evans, Universidad Iberoamericana León-México; José } \\
\text { Claudio Carrillo, Universidad de Guadalajara-México; Antonio Gómez } \\
\text { Nashiki, Universidad de Colima-México; Claudia Saucedo Ramos, Fa- } \\
\text { cultad de Estudios Superiores Iztacala, Universidad Nacional Autóno- } \\
\text { ma de México-México. }\end{array}$ \\
\hline $\begin{array}{l}\text { 12. La educación } \\
\text { básica en el Norte } \\
\text { ante el confinamien- } \\
\text { to sanitario } \\
\quad 112 \text { minutos }\end{array}$ & $\begin{array}{l}2 \text { de junio } \\
\text { de } 2020\end{array}$ & $\begin{array}{l}\text { Sandra Vega Villarreal, Universidad Pedagógica Nacional del Estado de } \\
\text { Chihuahua-México; María Soledad Ramírez Montoya, Instituto Tecno- } \\
\text { lógico de Monterrey-México; José Ángel Vera Noriega, Centro de In- } \\
\text { vestigación en Alimentación y Desarrollo, A. C.-México; Sergio Gerardo } \\
\text { Málaga Villegas, Universidad Autónoma de Baja California-México. }\end{array}$ \\
\hline $\begin{array}{l}\text { 13. Consecuencias } \\
\text { educativas de la } \\
\text { pandemia COVID-19 } \\
\text { en los estados de } \\
\text { Michoacán, Jalisco, } \\
\text { Aguascalientes y San } \\
\text { Luis Potosí } \\
\quad 116 \text { minutos }\end{array}$ & $\begin{array}{l}12 \text { de junio } \\
\text { de } 2020\end{array}$ & $\begin{array}{l}\text { Martha Vergara Fregoso, Universidad de Guadalajara-México; Ana } \\
\text { María Méndez Puga, Universidad Michoacana de San Nicolás de Hidal- } \\
\text { go-México; José Matías Romo Martínez, Universidad Pedagógica Na- } \\
\text { cional, Unidad o11, Aguascalientes-México; Yolanda López Contreras, } \\
\text { Directora de Unidades Universidad Pedagógica Nacional-México. }\end{array}$ \\
\hline $\begin{array}{l}\text { 14. La evaluación del } \\
\text { aprendizaje en tiem- } \\
\text { pos de pandemia: } \\
\text { entre el control y la } \\
\text { equidad } \\
\quad 120 \text { minutos }\end{array}$ & $\begin{array}{l}18 \text { de junio } \\
\text { de } 2020\end{array}$ & $\begin{array}{l}\text { María Isabel Arbesú García, Universidad Autónoma Metropolitana, } \\
\text { Unidad Xochimilco-México; Graciela González Juárez, Escuela Na- } \\
\text { cional de Enfermería y Obstetricia, Universidad Nacional Autónoma } \\
\text { de México-México; Daniel González Lomelí, Universidad de Sonora- } \\
\text { México; Arcelia Martínez Bordón, Universidad Iberoamericana-México, } \\
\text { Jaime Ricardo Valenzuela González, consultor independiente y colabo- } \\
\text { rador del Instituto Tecnológico y de Estudios Superiores de Monterrey- } \\
\text { México. }\end{array}$ \\
\hline
\end{tabular}




\begin{tabular}{|c|c|c|}
\hline $\begin{array}{l}\text { 15. Tensiones entre } \\
\text { educación y edu- } \\
\text { cación inclusiva. } \\
\text { Desafíos a partir del } \\
\text { COVID-19 } \\
\quad 152 \text { minutos }\end{array}$ & $\begin{array}{l}23 \text { de junio } \\
\text { de } 2020\end{array}$ & $\begin{array}{l}\text { Carlos Skliar, Facultad Latinoamericana de Ciencias Sociales-Argenti- } \\
\text { na; Walter Kohan, Universidad del Estado de Río de Janeiro-Brasil; Ge- } \\
\text { rardo Echeita Sarrionandia, Universidad Autónoma de Madrid-España; } \\
\text { Rodolfo Cruz Vadillo, Universidad Popular Autónoma del Estado de } \\
\text { Puebla-México; Zardel Jacobo, Facultad de Estudios Superiores Iztaca- } \\
\text { la, Universidad Nacional Autónoma de México-México. }\end{array}$ \\
\hline $\begin{array}{l}\text { 16. La importancia } \\
\text { de la educación en } \\
\text { saberes disciplinares } \\
\text { para comprender y } \\
\text { enfrentar la pande- } \\
\text { mia COVID-19 y las } \\
\text { nuevas realidades } \\
\text { sociales } \\
\quad 126 \text { minutos }\end{array}$ & $\begin{array}{l}29 \text { de junio } \\
\text { de } 2020\end{array}$ & $\begin{array}{l}\text { Ana María Méndez Puga, Universidad Michoacana de San Nicolás de } \\
\text { Hidalgo-México; Alfredo García Martínez, Facultad de Artes Plásticas } \\
\text { de la Universidad Veracruzana-México; Santiago Alonso Palmas Pérez, } \\
\text { Universidad Autónoma Metropolitana, Unidad Lerma-México, Sergio } \\
\text { Correa Gutiérrez, Unidad Académica Multidisciplinaria de Ciencias, } \\
\text { Educación y Humanidades de la Universidad Autónoma de Tamaulipas- } \\
\text { México; Felipe Martínez Rizo, consultor independiente-México. }\end{array}$ \\
\hline
\end{tabular}

Fuente: COMIE (2020b).

Anexo 3

Seminario "Cómo prepararse ante contingencias en instituciones educativas" de ANUIES, CUDI y RedLaTE

\begin{tabular}{|c|c|c|}
\hline Sesión & Fecha/duración & Instituciones participantes \\
\hline 1. & $\begin{array}{l}12 \text { de marzo de } \\
2020 \\
80 \text { minutos }\end{array}$ & $\begin{array}{l}\text { El uso de la tecnología educativa en momentos de crisis: de la visión remedial a la } \\
\text { incorporación cotidiana, María Luisa Zorrilla Abascal, Universidad Autónoma del } \\
\text { Estado de Morelos; Plan de Continuidad Académica de la Universidad Autónoma } \\
\text { de Baja California, Yessica Espinosa Díaz, Universidad Autónoma de Baja Cali- } \\
\text { fornia; Perspectiva de Microsoft México, Ediel Cortés, Consultor de Tecnología } \\
\text { en Educación. Microsoft México; Perspectiva de Google for Education, Juan José } \\
\text { Arroyo Co-founder ApiSec Cloud Services CEO, ieducando México; Laura Cama- } \\
\text { cho, Dirección de Pedagogía ieducando México partners de Google for Educa- } \\
\text { tion. }\end{array}$ \\
\hline 2. & $\begin{array}{l}19 \text { de marzo de } \\
2020 \\
94 \text { minutos }\end{array}$ & $\begin{array}{l}\text { Plan de contingencia en las IES frente al COVID-19, Luis Alberto Gutiérrez Díaz de } \\
\text { León, Investigador de la Universidad de Guadalajara; Acciones en la Universidad } \\
\text { Autónoma Metropolitana frente al COVID-19, Max de Mendizábal, Director de } \\
\text { Tecnologías de la Información de la Universidad Autónoma Metropolitana; Solu- } \\
\text { ciones AWS para la Continuidad de Operaciones, Carlos González Villegas, Geren- } \\
\text { te de los territorios de Puerto Rico y Mexico del Sector Publico de AWS. }\end{array}$ \\
\hline 3. & $\begin{array}{l}26 \text { de marzo de } \\
2020 \\
123 \text { minutos }\end{array}$ & $\begin{array}{l}\text { Plan de Continuidad Académica del Instituto Tecnológico y de Estudios Superiores } \\
\text { de Monterrey; Plan de Continuidad Académica del Instituto Politécnico Nacional; } \\
\text { Plan de Continuidad Académica de la Universidad ORT México. }\end{array}$ \\
\hline 4 . & $\begin{array}{l}16 \text { de abril de } \\
2020 \\
105 \text { minutos }\end{array}$ & $\begin{array}{l}\text { Plan de contingencia en el Instituto Tecnológico Autónomo de México frente al CO- } \\
\text { VID-19, Dra. Ana Lidia Franzoni Velázquez, Instituto Tecnológico Autónomo de } \\
\text { México; Plan de contingencia en la Universidad La Salle Ciudad de México, Dra. } \\
\text { María del Carmen de Urquijo Carmona, Universidad La Salle Ciudad de México; } \\
\text { Higiene Digital: Recomendaciones generales de Ciberseguridad, Mtro. Erick Yesser } \\
\text { Rodríguez Arreola, Universidad Autónoma de Chihuahua. }\end{array}$ \\
\hline
\end{tabular}




\begin{tabular}{|c|c|c|}
\hline 5. & $\begin{array}{c}23 \text { de abril de } \\
2020 \\
74 \text { minutos }\end{array}$ & $\begin{array}{l}\text { Plan de contingencia Campus Virtual UNAM, Dra. Concepción Barrón Tirado y Dr. } \\
\text { Jorge León Martínez, Universidad Nacional Autónoma de México; Plan de con- } \\
\text { tingencia en el Tecnológico Nacional de México, Dra. Mireya Saraí García Vázquez, } \\
\text { Tecnológico Nacional de México; Soluciones Cisco ante la contingencia por el CO- } \\
\text { VID-19, Manuel Acosta, Cisco Systems de México. }\end{array}$ \\
\hline 6. & $\begin{array}{c}30 \text { de abril de } \\
2020 \\
99 \text { minutos }\end{array}$ & $\begin{array}{l}\text { Plan de contingencia en la Universidad Pedagógica Nacional, Mtro. Víctor Álvarez } \\
\text { Castorela, Universidad Pedagógica Nacional; Plan de contingencia en la Univer- } \\
\text { sidad Autónoma de Yucatán, Mtro. Sergio Antonio Cervera Loeza, Universidad } \\
\text { Autónoma de Yucatán; Herramientas alternativas de aprendizaje ante contextos } \\
\text { de distanciamiento social, Ricardo Vázquez Gutiérrez, Co-fundador Oruka. }\end{array}$ \\
\hline 7. & $\begin{array}{c}7 \text { de mayo de } \\
2020 \\
73 \text { minutos }\end{array}$ & $\begin{array}{l}\text { Plan de contingencia en la Universidad Autónoma del Carmen, Dr. José Antonio } \\
\text { Ruz Hernández, Universidad Autónoma del Carmen y Mtra. Erika Sánchez Cha- } \\
\text { blé, Coordinación General de Tecnologías de la Información y Comunicaciones, } \\
\text { Universidad Autónoma del Carmen; Plan de contingencia en la Universidad Vera- } \\
\text { cruzana, Mtro. Juan Carlos Jiménez Márquez, Universidad Veracruzana; Plan de } \\
\text { contingencia en la Universidad Autónoma de Nuevo León, Mtro. Joaquín Huante } \\
\text { Hernández, Universidad Autónoma de Nuevo León. }\end{array}$ \\
\hline 8. & $\begin{array}{c}14 \text { de mayo de } \\
2020 \\
109 \text { minutos }\end{array}$ & $\begin{array}{l}\text { Implementación de herramientas de proctoring en la Universidad Autónoma de } \\
\text { Chihuahua, Mtro. Miguel Ángel López Santillán, Universidad Autónoma de Chi- } \\
\text { huahua y Huber Macias, SEN3 Group; Implementación de herramientas de procto- } \\
\text { ring en el Instituto Tecnológico y de Estudios Superiores de Monterrey; Mtra. Sadie } \\
\text { Lissette Guerrero Solis, Instituto Tecnológico y de Estudios Superiores de Mon- } \\
\text { terrey; Implementación de herramientas de proctoring en el Instituto Politécnico } \\
\text { Nacional, Mtro. Chadwick Carreto Arellano, Instituto Politécnico Nacional. }\end{array}$ \\
\hline 9. & $\begin{array}{c}21 \text { de mayo de } \\
2020 \\
101 \text { minutos }\end{array}$ & $\begin{array}{l}\text { Implementación de herramientas de proctoring en la Universidad Internacional de } \\
\text { la Rioja, Dra. Julieta Palma Anda, Universidad Internacional de la Rioja en Mé- } \\
\text { xico y Mtro. David Marina Osle, Universidad Internacional de de la Rioja; Imple- } \\
\text { mentación de herramientas de proctoring en Universidad Autónoma de Guerrero, } \\
\text { M.A. Tomás Iván Benítez Castro, Universidad Autónoma de Guerrero, Dr. Efrén } \\
\text { Marmolejo Valle, Universidad Autónoma de Guerrero. }\end{array}$ \\
\hline 10. & $\begin{array}{c}28 \text { de mayo de } \\
2020 \\
93 \text { minutos }\end{array}$ & $\begin{array}{l}\text { Plan de contingencia en la Universidad de las Américas Puebla, Mtro. Fernando } \\
\text { Thompson de las Rosa, Director General de Tecnologías de la Información de la } \\
\text { Universidad de las Américas Puebla (UDLAP); Programa académico de Question- } \\
\text { Pro, Emiliano Orduña, Academic Project Lead, Latin America QuestionPRO. }\end{array}$ \\
\hline 11. & $\begin{array}{l}4 \text { de junio de } \\
2020 \\
98 \text { minutos }\end{array}$ & $\begin{array}{l}\text { Proyecto Emergente de Enseñanza Remota, Mtro. Max de Mendizábal, Director } \\
\text { de Tecnologías de la Información de la Universidad Autónoma Metropolitana; } \\
\text { Plan de contingencia en la Universidad Autónoma del Estado de México, M. en } \\
\text { T.E. Alberto Torres Gutiérrez, Director de Educación Continua y a Distancia de la } \\
\text { Universidad Autónoma del Estado de México; Servicios Coursera en tiempos de } \\
\text { contingencia por COVID-19, Mtro. Francisco Forero Bonell, Coursera. }\end{array}$ \\
\hline 12. & $\begin{array}{c}11 \text { de junio de } \\
2020 \\
113 \text { minutos }\end{array}$ & $\begin{array}{l}\text { Plan de contingencia en la Universidad de Guadalajara, Mtra. Guadalupe Cid, } \\
\text { Coordinación General de Servicios Administrativos e Infraestructura Tecnológica } \\
\text { de la Universidad de Guadalajara; Plan de contingencia en Instituto Tecnológico } \\
\text { de Sonora, Mtro. Mario Acosta Ramirez, Coordinador Oficial de Seguridad Infor- } \\
\text { matica del Instituto Tecnológico de Sonora; Plan de contingencia en la Universi- } \\
\text { dad Autónoma de Tamaulipas, Mtro. Carlos Enrique Portes Flores, Dirección de } \\
\text { Tecnologías de Información de la Universidad Autónoma de Tamaulipas y Mtro. } \\
\text { Marco Antonio Treviño Rodríguez, Director de Información Académica y Admi- } \\
\text { nistrativa de la Universidad Autónoma de Tamaulipas. }\end{array}$ \\
\hline
\end{tabular}




\begin{tabular}{|l|c|l|}
\hline 13. & $\begin{array}{c}18 \text { de junio de } \\
2020 \\
111 \text { minutos }\end{array}$ & $\begin{array}{l}\text { Plan de contingencia en la Universidad Anáhuac, Mtro. José Valdez, ClO de la Red } \\
\text { de Universidades Anáhuac; Plan de contingencia en El Colegio de México, Mtro. } \\
\text { Canek Ramírez, Coordinador de Servicios de Cómputo en EI Colegio de México; } \\
\text { Plan de contingencia en la Universidad de Colima, Mtro. José Contreras, Coordi- } \\
\text { nador General de Tecnologías de Información de la Universidad de Colima. }\end{array}$ \\
\hline 14. & $\begin{array}{c}25 \text { de junio de } \\
2020 \\
121 \text { minutos }\end{array}$ & $\begin{array}{l}\text { Acciones realizadas por las Instituciones de Educación Superior para mantener los } \\
\text { servicios educativos ante el COVID-19, así como contribuir a la salud y bienestar } \\
\text { de la sociedad, Dra. Carmen Enedina Rodríguez Armenta, Directora General de } \\
\text { Educación Superior Universitaria/SEP; Perspectiva ECOESAD/UANL ante la contin- } \\
\text { gencia por el COVID-19, Dra. Lucero Cavazos, Directora de Educación a Distancia } \\
\text { de la Universidad Autónoma de Nuevo León (ECOESAD); Perspectiva UDUAL ante } \\
\text { la contingencia por el COVID-19, Dr. Germán Ruiz Méndez, Direción General de } \\
\text { Televisión Educativa y Unión de Universidades de América Latina y el Caribe. }\end{array}$ \\
\hline 15. & 2 de julio de \\
2020 & $\begin{array}{l}\text { Perspectiva en la Educación Superior en España ante la contingencia por el CO- } \\
\text { VID-19, Dr. Faraón Llorens; Perspectiva en la Educación Superior en Argentina } \\
\text { ante la contingencia por el COVID-19, Mtro. Ernesto Chinkes; Perspectiva en la } \\
\text { Educación Superior en Cuba ante la contingencia por el COVID-19, Mtro Alain La- } \\
\text { madrid, Ministerio de Educación Superior en Cuba; Perspectiva en la Educación } \\
\text { Superior en México ante la contingencia por el COVID-19, Dra. Marina Vicario, Ins- } \\
\text { tituto Politécnico Nacional. }\end{array}$ \\
\hline
\end{tabular}

Fuente: ANUIES, ANUIES -TIC, CUDI y RedLaTE (2020). 